\title{
Mapeamento das características dinâmicas do nível freático do Sistema Aquífero Bauru como instrumento de gestão de recursos hídricos
}

\author{
Mapping groundwater dynamics characteristics \\ of Bauru Aquifer System as water management instrument \\ Rodrigo Lilla Manzione ${ }^{1}$ \\ 'Universidade Estadual Paulista "Júlio de Mesquita Filho" - UNESP, Avenida Vitalina Marcusso, 1.500, \\ CEP 19910-206, Ourinhos, SP, BR (rlmanzione@gmail.com) \\ Recebido em 09 de setembro de 2017; aceito em 02 de março de 2018
}

\begin{abstract}
Resumo
O objetivo deste trabalho foi mapear o tempo de resposta dos níveis freáticos de aquíferos. Para tanto, recorreu-se à extração de séries temporais de dados de monitoramento de níveis freáticos, precipitação e evapotranspiração potencial em uma área representativa do Sistema Aquífero Bauru (SAB) na região hidrográfica do Médio Paranapanema (UGRHI-17), situada no Estado de São Paulo. A partir desses dados utilizou-se o modelo Predefined Impulse Response Function in Continuous Time (PIRFICT), obtendo-se uma função impulso-resposta (IR) cujos parâmetros são interpolados por metodologia geoestatística. Os resultados apresentaram-se como importantes ferramentas de auxílio para os tomadores de decisão quanto à adoção de práticas de manejo de resíduos e efluentes, uso da água, planos de remediação e políticas públicas para a gestão dos recursos hídricos subterrâneos.
\end{abstract}

Palavras-chave: Séries temporais; Geoestatística; Monitoramento; Sistema Aquífero Bauru; Níveis freáticos.

\section{Abstract}

The aim of this study was to map the response time of water table depths. For this purpose, time series of groundwater, precipitation and potential evapotranspiration monitoring data were extracted in a representative area of Bauru Aquifer System (BAS), at Médio Paranapanema hydrographical region (UGRHI-17), located in São Paulo state, Brazil. From this data, impulse-response (IR) functions were obtained calibrating the so-called Predefined Impulse Response Function in Continuous Time (PIRFICT) model. Model parameters were interpolated using geostatistical methodology. The results presented themselves as important ancillary tools for decision makers in adopting waste and effluent management practices, water use, remediation plans, and public policies for water resource management.

Keywords: Time series; Geostatistics; Monitoring; Bauru Aquifer System; Water table depths. 


\section{INTRODUÇÃO}

Na era da tecnologia da informação (TI), a adequação de métodos e técnicas de extração e mineração de dados (data mining) é cada vez mais necessária para se disponibilizar informações ao usuário, bem como auxiliar a tomada de decisão pelos planejadores e gestores de um dado sistema. Desse modo, a quantidade de informações disponíveis aos usuários e aos tomadores de decisões fez com que, naturalmente, o interesse em maximizar o uso da informação fosse crescendo entre a comunidade científica e os usuários de TI, de modo que diversos ramos da análise estatística e da modelagem matemática de dados fossem popularizados (Dagan, 2002; Zhang e Zhang, 2004; Rubin, 2004; Renard, 2007; Manzione, 2014). Os cenários futuros sobre incertezas climáticas também impulsionaram a popularização e, principalmente, a interpretação de resultados de experimentos estocásticos (estatísticos e probabilísticos) (Pachauri e Meyer, 2015).

A gestão de recursos hídricos superficiais e subterrâneos requer o uso de técnicas de modelagem que reconheçam a variabilidade e a incerteza associadas aos elementos hidrológicos. Modelos matemáticos e estatísticos têm sido aplicados à descrição de fenômenos hidrológicos por se tratarem de abstrações da realidade encontrada. Trata-se de uma forma de representação de uma ou de todas as propriedades de um fenômeno, sistema ou objeto. A modelagem em geral tem como propósito compreender melhor a resposta de processos a partir de observações realizadas, ou mesmo deduzir efeitos (Tucci, 2005; Pappenberger e Beven, 2006). A construção de um modelo é uma tentativa de ganho em conhecimento sobre determinado fenômeno. Um modelo nada mais é do que uma abstração da realidade. À medida que o conhecimento sobre um processo se expande, a complexidade dos modelos tende a aumentar. $\mathrm{O}$ uso de um modelo é justificado quando se pretende representar um sistema e a forma como ocorrem as modificações nesse sistema (Tucci, 2005). Por outro lado, a utilização de um modelo é útil para a realização de prognósticos e projeções futuras de determinadas situações, seja por meio do uso de abordagem determinísticas, probabilísticas (por exemplo, simulações), ou não probabilísticas (por exemplo, lógica fuzzy). Com ênfase na abordagem probabilística, a utilização de modelos estocásticos apresenta soluções importantes na estimativa de incertezas (Heuvelink e Pebesma, 1999). No que se refere aos processos hidrológicos em estudo, esses modelos são capazes de inferir sobre uma dada distribuição espaçotemporal e avaliar a sensibilidade (incertezas) das variáveis consideradas como estocásticas.

Portanto, no que se refere às medidas de incerteza, uma avaliação do risco relacionada pelas avaliações da vulnerabilidade intrínseca e do potencial de causar danos advindos de fontes pontuais ou difusas, quando associada às ações de gerenciamento, tais como as outorgas de irrigação e o dimensionamento do volume de água dos sistemas aquíferos, pode ser incluída no planejamento dos recursos hídricos, das vazões de poços para abastecimento urbano ou até mesmo no dimensionamento de complexos industriais. A avaliação da variabilidade espaçotemporal dos fenômenos hidrológicos é composta por elementos que permitem analisar a dinâmica do ciclo da água. Rubin (2004) apresentou uma série de soluções utilizando modelos estocásticos para problemas em águas subterrâneas relacionados à caracterização de processos, à modelagem de fluxo, ao transporte de solutos em meios heterogêneos e à quantificação de incertezas.

No que se refere à inferência sobre a variabilidade espaçotemporal, faz-se importante considerar sobre uma particularidade quanto à obtenção de dados. Quando coletado em tempo real, por sensores remotos e/ou em redes de monitoramento geoespaciais, o componente geográfico, capaz de revelar padrões não discerníveis por métodos estatísticos tradicionais, deve ser considerado. Por outro lado, quando os dados são registrados continuamente por meio de instrumentos tipo datalogger, com uma determinada frequência amostral, também se adiciona o componente temporal, presente em dados de monitoramento, por exemplo. Independentemente da forma como os dados são coletados, o monitoramento das águas subterrâneas é instrumento fundamental para a avaliação das condições em que esse meio natural se encontra e, posteriormente, pode ser utilizado como base ao tomar medidas preventivas e/ou proativas para manutenção da qualidade das águas subterrâneas e das reservas dos aquíferos, buscando desenvolver o uso sustentável junto a uma ação integrada de gerenciamento (Mestrinho, 2008). O monitoramento dos aquíferos é instrumento da Política Nacional dos Recursos Hídricos definido pela Lei no 9.433, de 1997, para apoiar os planos de recursos hídricos, a outorga para os diferentes usos da água e o seu enquadramento em classes. Portanto, a principal característica de uma série temporal de monitoramento é a dependência entre as observações realizadas. Assim sendo, a análise de séries temporais apresenta técnicas capazes de analisar a dependência temporal entre intervalos amostrais (Hipel e McLeod, 1994).

Nesses termos, Yi e Lee (2004) utilizaram modelos de séries temporais para preencher e completar séries irregulares de carga hidráulica a partir de dados de precipitação. Hatch et al. (2006) quantificaram interações entre águas subterrâneas e águas superficiais para estimativas da recarga, enquanto Von Asmuth et al. (2008) fizeram uma avaliação de como múltiplos estresses (escoamento de base, bombeamentos, vegetação, clima, barragens) influenciam no comportamento dos níveis das águas subterrâneas. Peterson e Western (2014) utilizaram modelos mais complexos de séries temporais para capturar comportamentos não lineares da drenagem do solo e Hocking e Kelly (2016), por sua vez, calcularam o tempo de resposta da recarga em aquíferos livres a partir da precipitação incidente. A análise de séries temporais, associada à análise espacial de dados geográficos, permite acessar as dimensões temporais espacial da 
variabilidade do fenômeno em estudo, nomeadamente oscilações dos níveis freáticos (Manzione et al., 2012).

Assim, o objetivo deste trabalho foi mapear o tempo de resposta das águas subterrâneas. Para tanto, recorreu-se à extração de uma série de dados de monitoramento de estações agro-hidrometeorológico (que fazem medição de nível freáticos, precipitação e evapotranspiração potencial) em uma área representativa do Sistema Aquífero Bauru (SAB) na UGRHI-17. A partir desses dados utilizou-se o modelo Predefined Impulse Response Function in Continuous Time (PIRFICT), obtendo-se uma função impulso-resposta (IR) cujos parâmetros foram interpolados por metodologia geoestatística. Desse modo, espera-se entender os mecanismos de oscilação das águas subterrâneas em aquíferos livres afetados, principalmente, pela sazonalidade do regime pluviométrico e, particularmente, inferir sobre a dinâmica do referido sistema aquífero analisado.

\section{MATERIAIS E MÉTODOS}

\section{Área de estudo}

A Estação Ecológica de Santa Bárbara (EEcSB) está localizada às margens da Rodovia SP-261 (km 58), coordenadas
2248'59" sul e 49 $14^{\circ}$ '12" oeste, em Águas de Santa Bárbara, São Paulo (Figura 1). A área foi regulamentada como unidade de conservação pelo Decreto $\mathrm{n}^{\circ} 22.337$, de 07 de junho de 1984, que delimitou uma área de 4.371 ha dentro dos limites da Floresta Estadual de Santa Bárbara.

A EEcSB é ocupada por vegetação nativa, campos antrópicos, vegetação em estágio de regeneração, mata ciliar e áreas de reflorestamento com pinus e eucalipto. $\mathrm{O}$ manejo na vegetação é constante, tanto para fins de produção florestal para pesquisa como para reverter a invasão do pinus sobre a vegetação nativa. Segundo Melo e Durigan (2011), a EEcSB protege amostras importantes das fisionomias abertas do Cerrado e uma pequena porção representativa da Floresta Estacional Semidecidual, estando inserida em uma região definida como de nível de prioridade cinco (considerando oito como nível máximo) para estabelecimento de ações de restauração interligando fragmentos de vegetação nativa e com prioridade máxima para inventários biológicos.

As formações geológicas aflorantes na região são os arenitos do Grupo Bauru, Formações Adamantina e Marília, com predomínio da formação Adamantina na EEcSB (CPRM, 2006; Melo e Durigan, 2011). Conforme o Mapa Geológico do Estado de São Paulo (IPT, 1981), a Formação Adamantina é caracterizada como depósitos fluviais com predominância
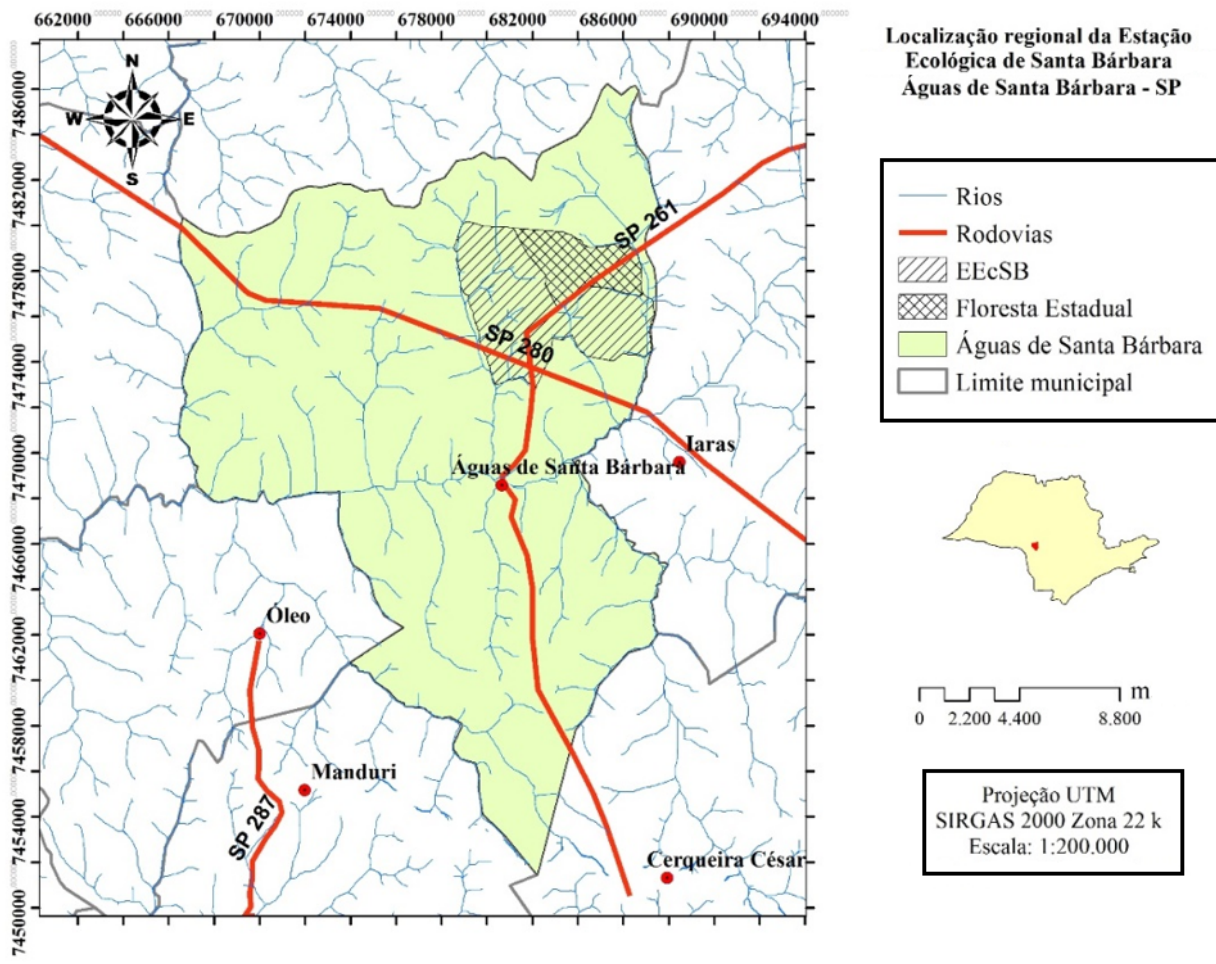

Projeção UTM

SIRGAS 2000 Zona $22 \mathrm{k}$

Escala: 1:200.000

Fonte: Santarosa (2016)

EEcSB: Estação Ecológica de Santa Bárbara.

Figura 1. Localização da Estação Ecológica de Santa Bárbara e da Floresta Estadual nos limites do município de Águas de Santa Bárbara, São Paulo. 
de arenitos finos, podendo apresentar cimentação e nódulos carbonáticos, com lentes de siltito arenosos e argilosos, ocorrendo em bancos maciços. Estratificação plano-paralela e cruzada de pequeno e médio porte. A Formação Marília apresenta arenitos de granulação fina a grossa, compreendendo bancos maciços com tênues estratificações cruzadas de médio porte, incluindo lentes e intercalações subordinadas a siltitos, argilitos e arenitos muitos finos com estratificação plano-paralela, frequentes níveis rudáceos e presença comum de nódulos carbonáticos. Existe ainda a ocorrência da Formação Serra Geral, de origem ígnea, extrusiva, formada por derrames de lavas basálticas, originando rochas de granulação muito fina, com coloração cinza a preta.

A EEcSB encontra-se localizada na Bacia Sedimentar do Paraná (morfoestrutura) e no Planalto Ocidental Paulista (morfoescultura), conforme descrito em Ross e Moroz (1996), possuindo, predominantemente, como formas de relevo colinas amplas e baixas, com altimetria em torno de 600 a $680 \mathrm{~m}$. As formas de relevo, combinadas a formações rochosas predominantes, possibilitam a ocorrência de Latossolos Vermelhos (LV56), Argissolos VermelhosAmarelo e Vermelho (PVA10) - eutróficos e distroférricos com textura arenosa/média - e Nitossolos (NV1) eutroférricos com textura argilosa (Oliveira et al., 1999).

O clima característico da região, segundo a classificação de Köeppen, é Cwa ou tropical subúmido (clima quente com inverno seco), apresentando temperaturas de $16^{\circ} \mathrm{C}$ no mês mais frio e de $23^{\circ} \mathrm{C}$ no mês mais quente (CEPAGRI, 2016).
As precipitações anuais estão em torno de 1.000 e 2.086 $\mathrm{mm}$, podendo chegar a $30 \mathrm{~mm}$ mensais no inverno. A temperatura média anual está em torno de $18^{\circ} \mathrm{C}$, com máxima entre 22 e $30^{\circ} \mathrm{C}$ em janeiro e mínima de $18^{\circ} \mathrm{C}$ no mês mais frio (Melo e Durigan, 2011).

\section{Dados disponíveis}

Foram utilizados dados de altura do lençol freático — monitorada mensalmente em 32 poços instalados próximos às principais drenagens da EEcSB - entre julho de 2014 e setembro de 2016 (Figura 2).

Também foram utilizados dados climatológicos de precipitação e de evapotranspiração potencial coletados, calculados e armazenados por uma estação climatológica compacta automática (ECA) instalada na área de estudo em julho de 2014. A ECA coleta e armazena dados diários, em frequência horária, de velocidade e direção do vento, radiação solar, temperatura, umidade relativa e precipitação.

Além disso, a evapotranspiração potencial de referência $\left(E T_{\text {ref }}\right)$ é calculada automaticamente pela ECA utilizando o método padronizado da American Society of Civil Engineers (ASCE) conforme a Equação 1:

$E T_{\text {ref }}=\frac{0.408 \Delta\left(R_{n}-G\right)+\gamma \frac{C_{n}}{T+273} u_{2}\left(e_{s}-e_{a}\right)}{\Delta+\gamma\left(1+C_{d} u_{2}\right)}$

Em que:

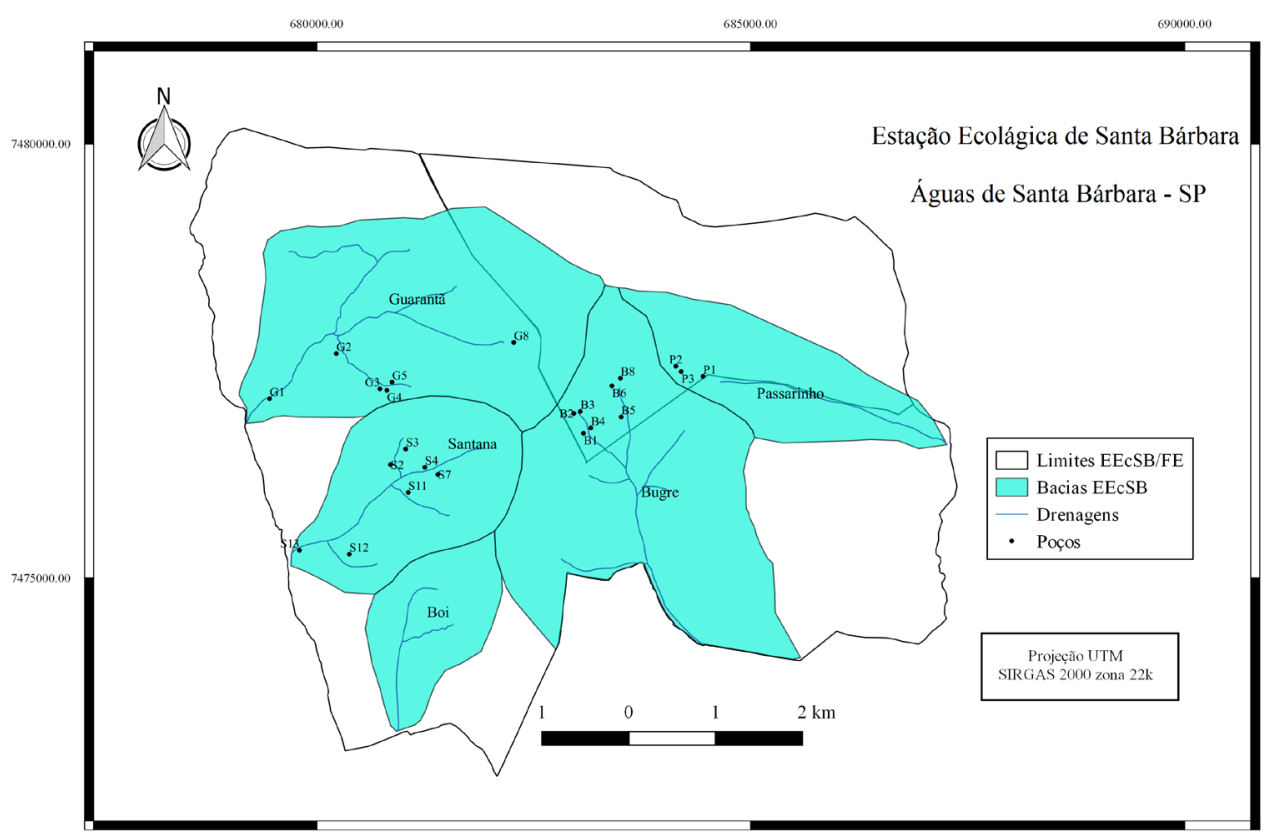

EEcSB: Estação Ecológica de Santa Bárbara; FE: Floresta Estadual.

Figura 2. Localização dos poços de monitoramento nas principais bacias hidrográficas da Estação Ecológica de Santa Bárbara e da Floresta Estadual. 
$R_{\mathrm{n}}=\mathrm{o}$ saldo de radiação na superfície $\left(\mathrm{MJ} \cdot \mathrm{m}^{-2} \cdot \mathrm{dia}^{-1}\right)$;

$G=$ o fluxo de calor do solo (MJ.m ${ }^{-2} \cdot$ dia $\left.^{-1}\right)$;

$\mathrm{T}=$ a temperatura média do ar a $2 \mathrm{~m}$ de altura $\left({ }^{\circ} \mathrm{C}\right)$;

$u_{2}=$ a velocidade média do vento a $2 \mathrm{~m}$ de altura $\left(\mathrm{m} . \mathrm{s}^{-1}\right)$;

$e_{\mathrm{s}}=$ a saturação de vapor média a $2 \mathrm{~m}$ de altura $(\mathrm{kPa})$;

$e_{\mathrm{a}}=$ a pressão média de vapor atual $\left(\mathrm{kPa} .{ }^{\circ} \mathrm{C}^{-1}\right)$;

$\gamma=$ a constante psicrométrica $\left(\mathrm{kPa} .{ }^{\circ} \mathrm{C}^{-1}\right)$;

$C_{\mathrm{n}}=$ o numerador constante para o tipo de referência e intervalo de cálculo;

$C_{\mathrm{d}}=$ o numerador constante para o tipo de referência e intervalo de cálculo.

Adotou-se como referência a vegetação rasteira (gramado). Basicamente, os parâmetros $C_{\mathrm{n}}$ e $C_{\mathrm{d}}$ são simplificações de termos da equação ASCE-Penman Monteith (Jensen et al., 1990), visando a uma estimativa mais universal da evapotranspiração potencial e demais computações a respeito da demanda hídrica de campos vegetados (Allen et al., 1998).

\section{Modelagem dos dados}

\section{Modelagem das séries temporais de monitoramento}

A partir das séries temporais de monitoramento dos níveis freáticos, de precipitação e de evapotranspiração potencial, utilizou-se o modelo PIRFICT para modelagem dos dados, que é uma alternativa a modelos de função e transferência de ruído em intervalos de tempo discretos apresentada por Von Asmuth et al. (2002).

Pelo modelo PIRFICT, o pulso em bloco de entrada é transformado em série de saída por uma função de transferência em tempo contínuo. Os coeficientes dessa função não dependem da frequência de observação. Após selecionar uma função IR de Pearson tipo III (Abramowitz e Stegun, 1965) que melhor se ajuste ao sistema e represente o processo físico em questão, a série temporal com as observações disponíveis tem de ser transformada em série contínua. A opção por esse tipo de função se dá por sua natureza flexível, ajustando-se a uma grande gama de respostas hidrológicas. No intuito de se caracterizar a variabilidade da precipitação e da evapotranspiração, recorre-se a um simples, porém eficiente, procedimento de estimativa da intensidade de precipitação excedente (ou déficit, se for o caso) e sua amplitude anual. A equação da função IR e seus parâmetros apresentam o sentido físico embutido na relação dinâmica entre precipitação e resposta no aquífero, como descrito por Von Asmuth e Knotters (2004). Assumindo-se linearidade, a componente determinística da dinâmica do lençol freático é completamente descrita pelos momentos da função IR. Nesse caso, os parâmetros podem ser definidos segundo Von Asmuth et al. (2002) (Equação 2):
$\theta(t)=A \frac{a^{n} t^{n-1} e^{-a t}}{\Gamma(n)}$
$\varphi(t)=\sqrt{2 \alpha \sigma_{r}^{2} e^{-a t}}$

Em que:

$A, a, n=$ os parâmetros da curva ajustada;

$\Gamma(n)=$ a função Gamma;

$\alpha$ controla a taxa de decaimento de $\varphi(t)$;

$\sigma_{r}^{2}=$ a variância dos resíduos.

O parâmetro $A$ está relacionado com a resistência à drenagem (a área da função IR é igual à razão entre a altura média do lençol freático e a recarga média) e fornece informações quanto ao tempo de resposta do lençol freático diante de eventos de precipitação. Em outras palavras, é o tempo em dias com que determinada entrada de água no sistema leva para deixar de influenciar a oscilação do nível da água subterrânea. Além das condicionantes hidrológicas, a área e a forma da função IR também podem sofrer interferência de algumas características hidrogeológicas, como tipo de substrato, uso e cobertura do solo e espessura da zona não saturada, conforme referiram Manzione et al. (2010). Nesse contexto, após um evento de precipitação, pode-se exemplificar a seguinte situação: quando o comportamento da resistência ao fluxo próximo à drenagem mais próxima for baixo, o nível do lenço freático apresentará um rápido decréscimo $\mathrm{e}$, consequentemente, a área formada pelo comportamento da função IR será pequena. Da mesma forma, tal ocorrência reflete-se também na memória do sistema hidrogeológico em relação a um evento de precipitação, podendo ser pequena, como no exemplo anterior, ou grande, quando o maciço poroso for extenso e os níveis forem mais profundos. O parâmetro $a$ é determinado pelo coeficiente de armazenamento do solo (porosidade), e $n$, pelo tempo de convecção e dispersão da precipitação pela zona não saturada. As bases físicas são explicadas por funções de transferência de uma série de reservatórios lineares (Nash, 1958). Para propostas nas quais se pretende modelar a resposta de uma bacia de forma geral, a idealização da bacia como um reservatório de armazenamento linear é o mais elementar entre vários níveis de conceptualização que isso envolve (Bodo e Unny, 1987). O parâmetro $n$ demonstra o número de reservatórios e $a$ é igual ao inverso do coeficiente de reservatório normalmente usado. Como explicam Knotters e Bierkens (2000), um simples reservatório linear (PIII df $\operatorname{com} n=1$ ) é igual a um mero modelo físico de coluna de solo unidimensional, descartando o fluxo lateral e o funcionamento da zona não saturada. Von Asmuth e Knotters (2004) chamam a atenção para cuidados ao interpretar esses parâmetros da função IR quanto ao seu sentido físico no processo, uma vez que suas bases são empíricas.

Por outro lado, o modelo PIRFICT pode ser processado a partir de múltiplas séries de entrada, como precipitação, 
evapotranspiração, fluxo de base em rios, testes de bombeamento, intervenções antrópicas, inclusão de tendências, passos e não linearidades (Von Asmuth et al., 2008). Assim, a partir dessas características, as análises do modelo PIRFICT foram realizadas com o auxílio do software Menyanthes (Von Asmuth et al., 2012). A avaliação inicial dos ajustes do modelo PIRFICT se deu por meio do cálculo da variância explicada pelo modelo (em inglês: explained variance percentage - EVP) em porcentagem, que é a variância da série de nível freático menos a variância da série de resíduos dividida pela variância da série de nível freático e multiplicada por 100 (Von Asmuth, 2012). Também foram utilizados os valores da raiz do erro médio quadrático (RMSE) e da raiz da inovação média quadrática (RMSI) como medidas de precisão do modelo, conforme descrito por Von Asmuth e Bierkens (2005). Para informações completas sobre descrição, formulação, aplicações e casos de estudo do modelo PIRFICT utilizado nesta pesquisa, recomenda-se consulta aos estudos descritos no trabalho de Von Asmuth (2012).

\section{Análise geoestatística}

Manzione et al. (2012) apresentam uma extensão à análise de séries temporais utilizando o modelo PIRFICT, via integração da dimensão temporal com a dimensão espacial por meio da interpolação geoestatística de parâmetros do modelo. Neste estudo, realizou-se essa análise para o parâmetro do tempo de resposta do modelo PIRFICT. Seguiu-se a metodologia descrita em Journel e Huijbregts (1978), Isaaks e Srivastava (1989), Goovaerts (1997) e Yamamoto e Landim (2013): variografia, interpolação dos dados por krigagem ordinária e validação cruzada dos resultados.

\section{Análise estrutural dos dados geográficos (variografia)}

O estimador geoestatístico da dependência espacial dos dados, conhecido por semivariograma, pode ser estimado por meio da Equação 3:

$\gamma^{*}(h)=\frac{1}{2 N(h)} \sum_{i=1}^{N(h)}\left[Z\left(x_{i}\right)-Z\left(x_{i}+h\right)\right]^{2}$

Em que:

$N(h)=$ o número de pares de valores medidos $\mathrm{Z}\left(x_{i}\right), \mathrm{Z}\left(x_{i}\right.$ $+h$ ), separados por um vetor $h$ (Journel e Huijbregts, 1978).

O gráfico de $\gamma^{*}(h)$ versus os valores correspondentes de $h$, chamado semivariograma, é uma função do vetor $h$ e, portanto, depende de ambas, magnitude e direção de $h$, ilustrando a relação entre a variância das amostras e suas distâncias laterais.

Por intermédio dessa relação, a distância lateral entre as amostras pode ser estimada a fim de otimizar o número de amostras e sua variância. A distância na qual o semivariograma atinge um valor de estabilidade é nomeada alcance, sendo o limite da dependência espacial. A esse valor, próximo à variância dos dados, dá-se o nome de patamar. $\mathrm{O}$ alcance da dependência espacial representa a distância em que os pontos amostrais estão correlacionados entre si. Os pontos localizados em uma área de raio maior ao alcance são independentes, apresentando uma distribuição espacial aleatória e menos homogênea. Chama-se efeito pepita $\left(C_{0}\right)$ a medida em que a distância $(h)$ tende a zero e a variação geralmente se aproxima de um valor finito. Segundo Burgess e Webster (1980), esse é um importante parâmetro do semivariograma, já que representa a variação residual e aleatória, não removida por amostragens próximas.

Os estudos variográficos foram processados considerando a adoção das direções $0,45,90$ e $135^{\circ}$, de modo que fossem construídos semivariogramas direcionais e omnidirecionais. Os semivariogramas omnidirecionais são normalmente considerados em uma das duas, ou nas duas, seguintes situações:

- semivariogramas direcionais semelhantes (situação de isotropia representada por um único semivariograma omnidirecional);

- escassez de dados e/ou semivariogramas direcionais demasiado erráticos.

Em seguida, ambos os semivariogramas foram sobrepostos, para detectar ocorrências ou não de continuidade espacial desigual em uma ou mais direções. A elaboração desses semivariogramas foi precedida das considerações acerca da distância do campo amostral $(L)$, em que se utilizou o valor de $L / 2$ para cálculo do semivariograma experimental, dividido em 12 passos (lags), com tolerância angular de $22,5^{\circ}$. A seguir, os modelos teóricos esférico, exponencial e gaussiano foram ajustados, em busca de um modelo teórico que melhor se adequasse ao fenômeno estudado. Para isso, utilizou-se o cálculo do grau de dependência espacial (GDE), que se refere o uso do percentual da variância de $C_{0}$, proposto por Trangmar et al. (1985). Assim sendo, Cambardella et al. (1994) propuseram classificação de GDE pelos seguintes graus:

- $\mathrm{GDE} \leq 25 \%$, forte dependência espacial;

- $25 \leq \mathrm{GDE} \leq 75 \%$, moderada dependência espacial;

- $\mathrm{GDE} \geq 75 \%$, fraca dependência espacial.

Após encontrar o melhor ajuste ao semivariograma, ou se detecta uma situação de isotropia e o modelo contínuo (representado geometricamente por uma circunferência) pode ter por base o semivariograma omnidirecional, ou se detecta anisotropia e o modelo contínuo (representado geometricamente por uma elipse orientada com eixos segundo as direções de maior e menor continuidade) deve ter por base um modelo de semivariograma anisotrópico. Por outro 
lado, caso tenha sido detectada a presença de anisotropias, operam-se as possíveis correções, nomeadamente anisotropias geométrica, zonal ou mista (combinada). A função variográfica escolhida entre os modelos teóricos esférico, gaussiano, exponencial e linear testados foi aquela que apresentou melhor curva ajustada aos dados por mínimos quadrados ordinários.

\section{Interpolação dos dados}

A krigagem é o método de interpolação em geoestatística, sendo utilizada para obtenção de mapas espaciais. Por intermédio dela pode-se atingir um dos objetivos centrais dos estudos sobre variabilidade espacial, que é obter, a partir de observações pontuais, informações para grandes áreas com base nas observações da variável a ser estimada em locais não amostrados. Supondo-se que se queira estimar valores, $z^{*}$, para qualquer local, $x_{0}$, no qual não se tem valores medidos, assumindo estacionariedade de ordem 2 (a variância não pode aumentar indefinidamente) e que a estimativa deve ser uma combinação linear dos valores medidos, o estimador será conforme a Equação 4:

$Z^{*}\left(x_{0}\right)=\sum_{i=1}^{N} \lambda_{i} Z\left(x_{i}\right)$

Na qual:

$N=$ o número de valores medidos $z\left(x_{i}\right)$ envolvidos na estimativa, $\mathrm{e}$

$\lambda_{\mathrm{i}}=$ os pesos associados a cada valor medido, $z\left(x_{\mathrm{i}}\right)$, segundo Isaaks e Srivastava (1989).

Trata-se de um estimador linear ponderado que calcula o valor dos pesos pela estimativa da estrutura espacial da distribuição das variáveis, representadas por um semivariograma experimental. Os pesos são variáveis de acordo com a variabilidade espacial expressa no semivariograma, sendo nada mais que uma média móvel ponderada, tornando-se um interpolador ótimo devido à sua distribuição. Mas, para que o estimador seja ótimo, ele não pode ser tendencioso e deve ter variância mínima. A condição de não tendência significa que, em média, a diferença entre valores estimados e medidos para o mesmo ponto deve ser nula. A condição de variância mínima significa que, embora possam existir diferenças ponto por ponto entre os valores estimado e medido, elas devem ser mínimas. Essa interpolação estatística é essencialmente idêntica à regressão linear múltipla, com algumas diferenças quanto ao uso das matrizes empregadas para resolução dos sistemas (Isaaks e Srivastava, 1989). Entre os tipos de krigagem existentes, o usado neste trabalho foi a krigagem ordinária, que considera a média local dos valores nas estimativas para locais não amostrados no domínio da área de estudo. Da mesma forma, em Manzione et al. (2010) investigou-se tendência a partir de dados altimétricos oriundos de modelos digitais de elevação (MDE), mas sem maiores correlações.
Ressalta-se que, caso seja detectada a formação de deriva, deve-se recorrer à krigagem de tendência ou à própria krigagem ordinária com um pequeno raio de busca, descritas em (Goovaerts, 1997). Assim, avalia-se e compara-se o desempenho do modelo variográfico ajustado.

\section{Validação cruzada}

Os semivariogramas podem ser avaliados pela técnica conhecida como validação cruzada, que permite comparar o impacto dos diferentes modelos de semivariogramas sobre os resultados da interpolação, retirando os dados atuais e reestimando-os por dados dos vizinhos que permaneceram (Goovaerts, 1997). É importante que se tenha um meio para checar se o modelo ajustado é satisfatório ou não, bem como para validar o plano de krigagem antes do seu uso na construção de mapas. Embora esse procedimento possa ser efetuado pelo erro médio (ME - mean error), neste estudo foram adotados como melhor estimativa o procedimento de validação cruzada que apresenta média padronizada (MS - mean standardized) próxima de zero, o menor valor possível da raiz da média quadrática (RMS - root-mean-square), a média do erro predito padrão (ASE - average standard error) próxima da média padronizada e o valor da raiz da média quadrática padronizada (RMSS - root-mean-square standardized) próximo de 1. Por sua vez, o indicador RMSS fornece uma medida da acurácia dos valores interpolados: quando abaixo de 1, pode ser considerada subestimação, e quando acima, superestimação dos dados (Johnston et al., 2001).

\section{RESULTADOS E DISCUSSÕES}

\section{Identificação os tempos de resposta dos sistemas hidrogeológicos}

De forma geral, o ajuste do modelo PIRFICT para os 32 poços de monitoramento da EEcSB foi considerado bom, ou seja, com os valores médios de EVP superiores a 90\% (Tabela 1). Os valores de RMSE foram considerados baixos, com uma

Tabela 1. Estatísticas médias das calibrações do modelo Predefined Impulse Response Function in Continuous Time nos 32 poços de monitoramento entre 05 de setembro de 2014 e 02 de setembro de 2016.

\begin{tabular}{lccccc}
\hline & EVP & RMSE & RMSI & A & DP(A) \\
\hline Média & 93,38 & 0,10 & 0,09 & 300,50 & 113,52 \\
Mínimo & 75,88 & 0,04 & 0,01 & 104,70 & 35,00 \\
Máximo & 98,03 & 0,22 & 0,18 & 597,00 & 350,00
\end{tabular}

EVP: variância explicada pelo modelo (\%); RMSE: raiz do erro médio quadrático $(\mathrm{m})$; RMSI: raiz da inovação média quadrática $(\mathrm{m})$; NDL: nível da drenagem local (m); A: resistência à drenagem (dias); DP: desvio padrão. 
média de $10 \mathrm{~cm}$. O mesmo se aplica aos valores de RMSI, que variaram de $9 \mathrm{~cm}$, na média. Segundo Von Asmuth e Bierkens (2005), as inovações são uma maneira mais robusta de examinar os ajustes, já que calculam os erros médios entre um instante no tempo e o instante anterior.

O parâmetro do tempo de resposta indica a resistência à drenagem em dias, ou seja, o tempo que o nível freático leva para responder ao início de um impulso da chuva até que ele deixe de influenciar o lençol, por exemplo. A interpretação da função IR pode ser feita considerando que o momento e a intensidade do pico da curva referemse ao primeiro efeito da variável de entrada no sistema aquífero, seguido pelo decaimento do reservatório subterrâneo até o retorno à condição inicial estabelecida pelo nível da drenagem fixado pelo modelo. Assim, a área da curva refere-se ao tempo total que o efeito da precipitação interfere no sistema aquífero. Esses parâmetros mostraram resposta rápida do sistema, ou seja, pico da função IR logo nos primeiros meses após a precipitação e queda gradual da função ao longo do tempo. Por respostas lentas entendem-se memórias de alguns meses ou mesmo anos (Manzione et al., 2010). A Figura 3 mostra exemplos para as funções IR ajustadas para o pulso de precipitação nas quatro bacias estudadas.

As funções IR para a precipitação na Bacia do Guarantã têm o mesmo formato, com diferenças na intensidade e no tempo dos seus picos, seguidas de um decaimento exponencial. Assim, o poço G5 encontra-se mais distante das drenagens, sendo mais influenciado pela precipitação do que os poços G2, G3 e G4, que estão mais próximos do curso d'água. Refere-se que, nesses três poços, o pico da função IR encontra-se entre 2,5 e 3 (adimensional), enquanto no poço G5 o valor aproxima-se a 3,75. Esses picos acontecem cerca de 10 dias após os eventos de precipitação, decaindo ao longo do tempo, o que denota uma resposta rápida dos sistemas de águas subterrâneas locais. Nos poços mais próximos à drenagem, nota-se pequena atuação da precipitação, uma vez que existe uma influência hidrodinâmica da descarga que também contribui para alterar o comportamento dos níveis freáticos.

Por outro lado, na Bacia do Bugre os poços também apresentaram comportamento rápido, de modo que os picos são atingidos de forma quase instantânea, com exceção do poço B6, que apresentou foi levemente mais lento. Em geral, os picos são atingidos entre 0,80 e 2,05 . Por essas características, sugere-se que esses poços se encontravam próximos às drenagens e nascentes. $\mathrm{O}$ poço $\mathrm{B} 8$, entretanto, por se encontrar um pouco mais distante de drenagens e nascentes, apresentou valores da função IR mais elevados, visto que é pouco influenciado pelo Ribeirão do Bugre.

Os poços S2 e S3, que se encontram locados na Bacia do Santana, apresentam a maior profundidade de nível freático em relação à superfície, indicando mais resistência à drenagem. Portanto, todas as funções IR para aferir a influência da precipitação sobre os níveis freáticos da Bacia do Santana apresentaram resposta rápida. Entretanto, níveis mais profundos apresentam valores de IR maior do que 3,0. Já os poços S4 e S7 apresentaram valores de IR inferiores,
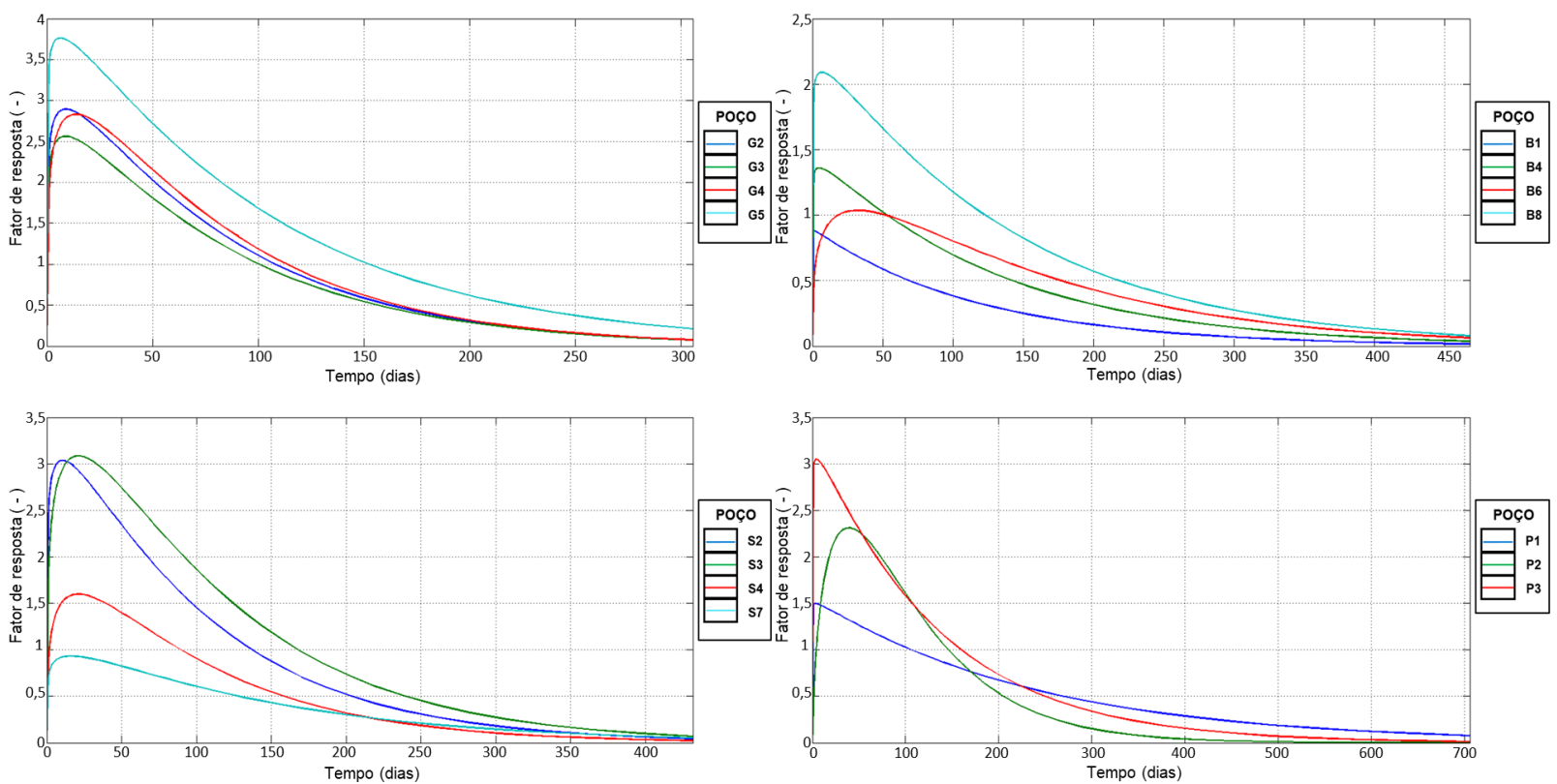

Figura 3. Exemplos de funções IR ajustadas para precipitação a partir da calibração do modelo Predefined Impulse Response Function in Continuous Time nas quatro bacias estudadas na Estação Ecológica de Santa Bárbara. 
uma vez que se encontram mais próximos ao curso d'água. Ressalta-se que o poço S7 apresentou níveis muito superficiais e passou boa parte do ano de 2016 com o entorno alagado, conforme verificado no local.

Assim como nas outras bacias, os poços da Bacia do Passarinho, designados como P1, P2 e P3, apresentaram respostas rápidas, sendo que os valores da função IR comportaram-se como sendo diretamente proporcionais à distância em relação à drenagem e à espessura da zona não saturada.

De forma geral, pode-se observar na EEcSB que os sistemas hidrogeológicos apresentam memória curta e respostas rápidas, variando em função da distância em relação à drenagem mais próxima e à espessura da zona não saturada, além de sofrerem forte influência da precipitação. Essa memória curta (ou resposta rápida) do $\mathrm{SAB}$ também foi verificada em formações sedimentares com níveis pouco profundos, como nas formações que recobrem a região de Planaltina, Distrito Federal (Manzione et al., 2010), e na Formação Botucatu, na região de Brotas, São Paulo (Manzione et al., 2017). O mesmo padrão também foi observado nos profundos Vertissolos da Austrália, nos quais Hocking e Kelly (2016) verificaram a relação direta entre a profundidade do nível freático e o tempo de resposta do aquífero diante dos eventos de precipitação e evapotranspiração. Nesse estudo, os referidos autores concluíram que quanto mais espessa a camada não saturada e maior for a distância da superfície até o nível d'água do poço, maior o tempo de resposta do sistema aquífero.

\section{Interpolação dos tempos de resposta}

O cálculo dos semivariogramas foi realizado utilizando como distância inicial o valor de metade do campo amostral considerado (8 km na direção leste-oeste). Foram divididos $4 \mathrm{~km}$ em 12 passos (lags) de 333,33 m. A partir desse valor inicial, foram testadas novas configurações de distância do campo amostral e do tamanho dos passos. Foram testados diferentes modelos teóricos ao semivariograma calculado para os 32 poços monitorados entre setembro de 2014 e agosto de 2016. A Tabela 2 apresenta os parâmetros do semivariograma ajustado para os tempos de resposta mapeados. A Figura 4 apresenta o semivariograma do ajuste realizado. Ressalta-se que, apesar da investigação, não foram encontradas variações direcionais na área de estudo que justificassem a adoção de outro semivariograma experimental que não fosse o omnidirecional.

O número de amostras selecionadas para cálculo afetou a modelagem da estrutura de dependência espacial dos dados, mas, mesmo com um número reduzido de amostras, pode-se modelar a variância dos dados em função da distância. Para tempo de resposta foi ajustado um modelo gaussiano com o valor total dos patamares encontrados; os efeitos pepita foram responsáveis por $22,87 \%$ do total da variância para o tempo de resposta, denotando forte dependência espacial (GDE $\leq 25 \%)$. O alcance para o tempo de resposta foi de $1.575,00 \mathrm{~m}$. O mapeamento dos tempos de resposta para a EEcSB pode ser visto na Figura 5. Como esperado, os tempos de resposta foram menores e mais

Tabela 2. Parâmetros do semivariograma ajustado para o tempo de resposta calculado a partir do modelo Predefined Impulse Response Function in Continuous Time.

\begin{tabular}{ccccccl}
\hline Variável & $\begin{array}{c}\text { Número de } \\
\text { lags }\end{array}$ & $\begin{array}{c}\text { Tamanhos da } \\
\text { lags }(\mathbf{m})\end{array}$ & Efeito Pepita & Patamar & Alcance $(\mathrm{m})$ & Modelo \\
\hline $\mathrm{A}$ & 12 & 181,00 & $4.860,00$ & $21.250,00$ & $1.575,00$ & Gaussiano \\
\hline
\end{tabular}

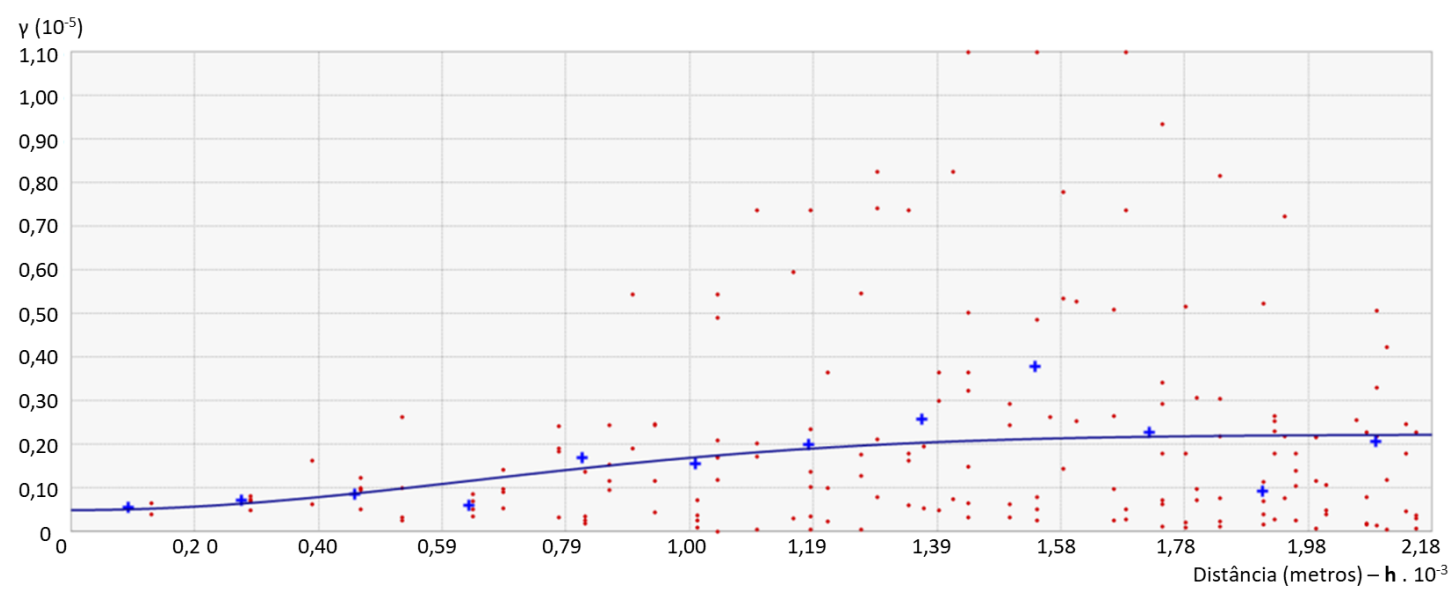

Figura 4. Semivariograma amostral e modelo teórico ajustado aos valores dos tempos de resposta calculados pelo modelo Predefined Impulse Response Function in Continuous Time. 
rápidos quanto mais próximo às drenagens estavam os poços. A espessura da zona não saturada nessas áreas é muito menor que nos divisores de água das bacias.

A Bacia do Passarinho revelou um padrão de resposta intermediário entre os valores mínimo (137,2 dias) e máximo (548,7 dias) interpolados por krigagem ordinária. Já a Bacia do Guarantã apresentou tendência de resposta maior em toda a sua cabeceira e nos cursos de primeira ordem. Essas áreas coincidem com as regiões mais elevadas da EEcSB. A Bacia do Bugre apresentou resposta mais rápida como um todo, enquanto a Bacia do Santana apresentou-se com respostas mais rápidas na cabeceira e mais lentas a jusante do canal principal. Esse cenário pode ser explicado pelo fato de a Bacia do Bugre escoar para uma área em que o fluxo superficial é mais característico, enquanto a Bacia do Santana escoa para uma área úmida represada pela SP-280 (Rodovia Presidente Castelo Branco), como apresentado na Figura 1.

A Tabela 3 apresenta os resultados da validação cruzada. Os valores da MS foram próximos a zero. Os valores da ASE foram próximos aos da RMS. Os valores da RMSS foram abaixo de 1, denotando a subestimação dos dados. Isso se deve ao efeito de suavização do interpolador por krigagem ordinária, que se refere ao valor médio, mas acaba apresentando erros nos valores mais extremos da distribuição normal dos dados (Yamamoto e Landim, 2013).
A partir da interpolação por krigagem, também é possível mapear os erros preditos padrão (SE) e verificar os locais em que a superfície de tendência calculada é mais confiável, ou seja, nos quais as incertezas associadas ao modelo de predição espacial são menores e, consequentemente, as inferências mais confiáveis (Figura 6). Os erros da interpolação são menores nas regiões próximas às drenagens, nas quais 0 volume de informação fornecida pelos poços de monitoramento é maior. Próximo aos divisores topográficos que limitam as Bacias do Santana, do Boi e do Guarantã as incertezas também foram baixas, com erros por volta de 90 dias. As áreas com maior incerteza são a jusante das Bacias do Bugre e do Boi, sobre as quais não há informação.

Essas informações sobre o tempo de resposta do lençol freático a partir de eventos de precipitação, assim como os erros envolvidos na predição, podem auxiliar planejadores e tomadores de decisão na gestão dos recursos hídricos.

Tabela 3. Validação cruzada para interpolação do tempo de resposta calculado a partir do modelo Predefined Impulse Response Function in Continuous Time.

\begin{tabular}{cccccc}
\hline Variável & ME & RMS & MS & RMSS & ASE \\
\hline A & $-6,420$ & 103,56 & $-0,0330$ & 0,97 & 101,92 \\
\hline
\end{tabular}

ME: erro médio; RMS: raiz da média quadrática; MS: média padronizada; RMSS: raiz da média quadrática padronizada; ASE: erro predito padrão médio.

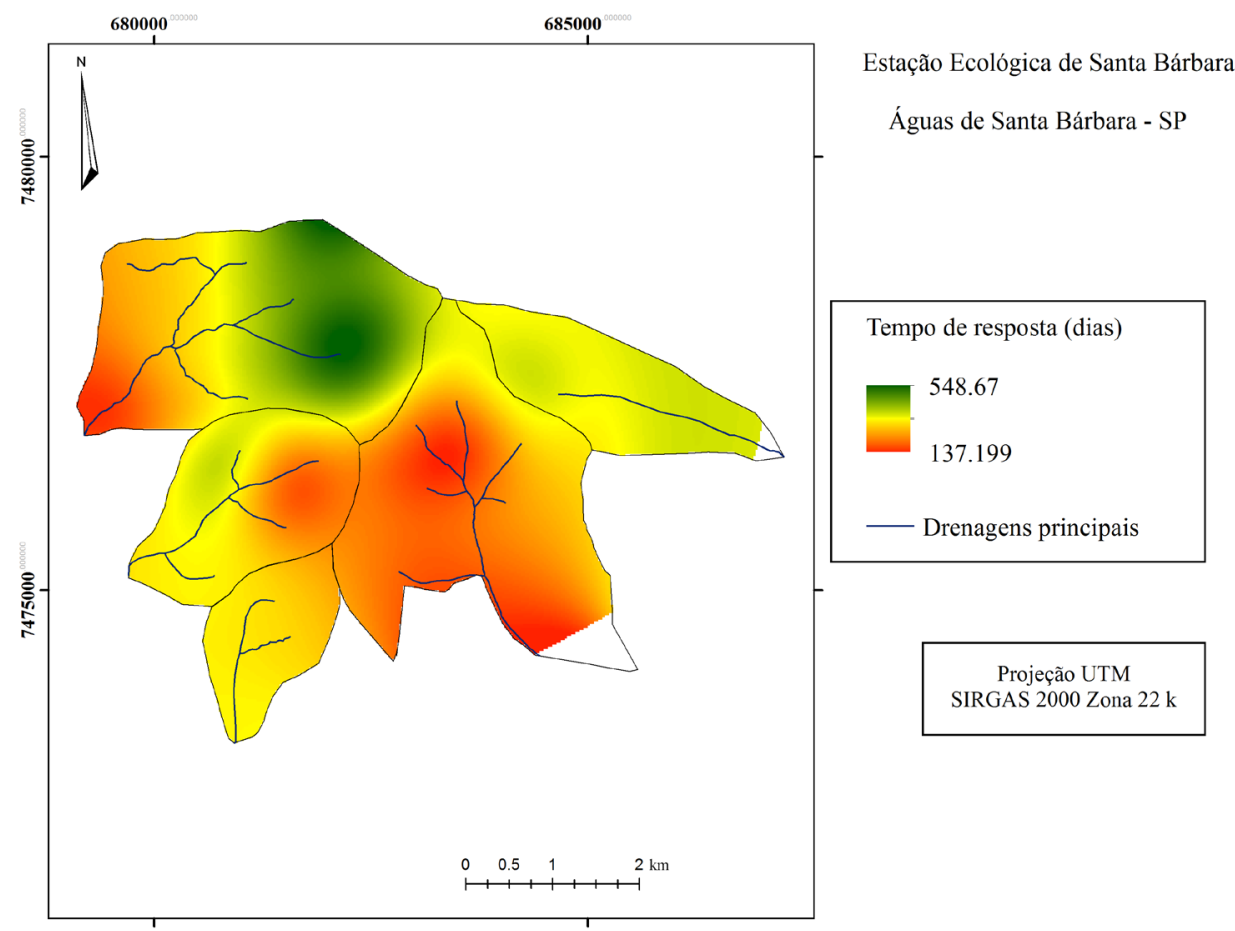

Figura 5. Mapa do tempo de resposta do lençol freático em função de eventos de precipitação interpolado por krigagem ordinária na Estação Ecológica de Santa Bárbara. 


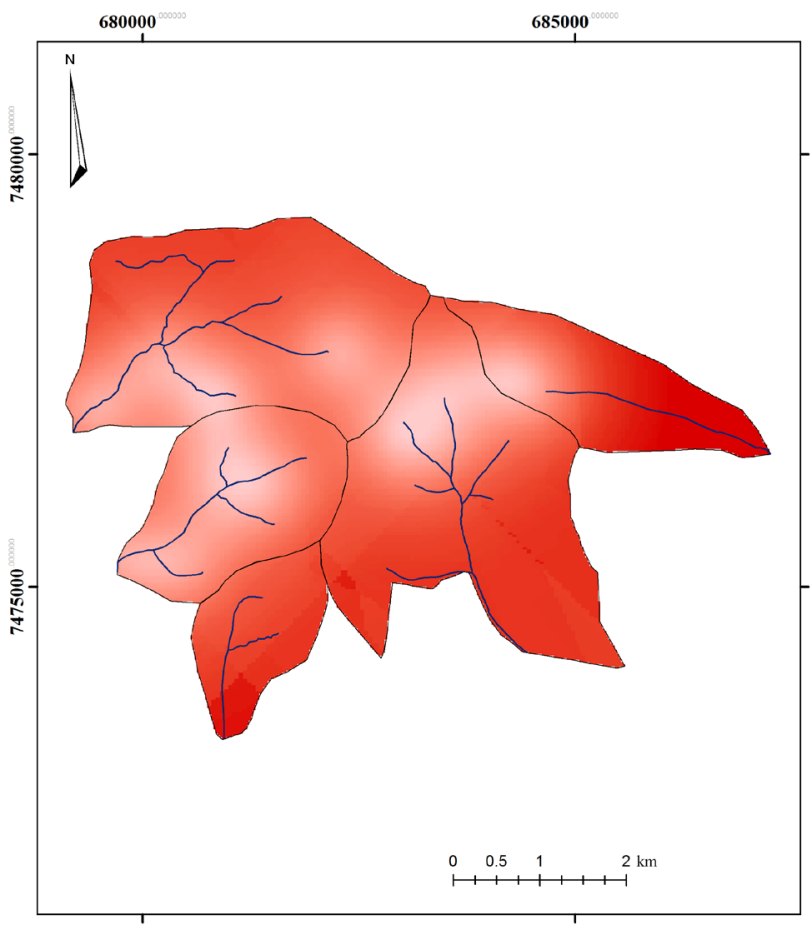

Estação Ecológica de Santa Bárbara

Águas de Santa Bárbara - SP

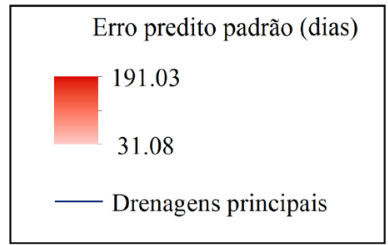

SIRGAS 2000 Zona $22 \mathrm{k}$

Figura 6. Mapa dos erros preditos padrão da interpolação do tempo de resposta do lençol freático em função de eventos de precipitação interpolado por krigagem ordinária na Estação Ecológica de Santa Bárbara.

A caracterização do meio físico, muitas vezes, não é suficiente para os processos cotidianos que envolvem a gestão, sendo necessária a caracterização da dinâmica do sistema aquífero para que se possa considerar a componente temporal na análise dos fenômenos reinantes. Dessa forma, é possível entender as respostas em relação a diferentes estímulos e otimizar a adoção de práticas de manejo de resíduos e efluentes, por exemplo, que dependem do tempo para que causem impactos no meio, assim como planos de remediação em caso de acidente ou mesmo contaminação.

Von Asmuth et al. (2008) apontam outros processos que podem influenciar a dinâmica dos níveis freáticos e que podem ser incluídos em análises sobre a dinâmica do sistema hidrogeológico, como escoamento de base, bombeamentos, vegetação, clima, barragens. A análise espacial aplicada à análise de bacias hidrográficas em escala local ou regional também pode ser aliada dos gestores, bem como os mapas gerados podem se servir de instrumentos para uso da água e políticas públicas quanto à capacidade suporte de áreas para atividades (Manzione et al., 2012).

Uma vez que os planos de bacia são instrumentos definidos pela Lei ${ }^{\circ}$ 9.433/97 para a gestão de recursos hídricos, a inclusão de mapas e suas respectivas medidas de incerteza deve ser praticada pelos órgãos responsáveis para adicionar novas métricas a essa equação de difícil solução. Manzione et al. (2006) mapearam tendências de elevação e rebaixamento de aquífero em bacias no Cerrado brasileiro buscando entender padrões que revelassem relações de oferta e demanda hídrica na região em função do clima. Manzione et al. (2010) elaboraram mapas de risco de níveis freáticos extremos para detectar possíveis áreas com lençol muito superficial ou muito profundo, o que em áreas agrícolas pode limitar as operações de campo. Os mapas deste estudo são um exemplo de como essas informações podem ser modeladas, descritas e interpretadas pelos gestores da água, buscando um uso racional e sustentável dos recursos hídricos subterrâneos e de suas interfaces com o clima.

\section{CONCLUSÕES}

A partir deste estudo pode-se demonstrar a aplicabilidade de modelos de séries temporais a dados de monitoramento de nível freático considerando os efeitos da sazonalidade. As principais conclusões reunidas a partir da modelagem dos dados do monitoramento agro-hidrometeorológico realizado foram:

- O modelo PRIFICT apresentou bons ajustes, identificando na EEcSB um sistema aquífero com memória 
curta, rápidas respostas aos estímulos climatológicos e forte influência sazonal, principalmente da precipitação;

- A análise espacial utilizando técnicas geoestatísticas revelou um padrão espacial do tempo de resposta gradual na área da EEcSB, variando suavemente no espaço, sem mudanças abruptas;

- Os resultados finais apresentados na forma de mapas auxiliam na interpretação dos dados de monitoramento, podendo ser incluídos nos planos de recursos hídricos como instrumento de gestão.

\section{AGRADECIMENTOS}

À Fundação de Amparo à Pesquisa do Estado de São Paulo (FAPESP), pelo auxílio financeiro para a realização desta pesquisa (Processo \# 2014/04524-7).

Ao Campus de Ourinhos da UNESP, pelo apoio logístico.

Aos alunos do Laboratório de Hidrologia e Hidrogeologia Operacional (LabH2O), do Campus de Ourinhos da UNESP, e aos funcionários do Instituto Florestal do Estado de São Paulo, pelo auxílio nos trabalhos de campo.

\section{REFERÊNCIAS}

Abramowitz, M., Stegun, I. A. (1965). Handbook of mathematical functions. Nova York: Dover Publications Inc.

Allen, R. G., Pereira, L. S., Raes, D., Smith, M. (1998). Crop evapotranspiration: guidelines for computing crop water requirements. Rome: FAO. (Irrigation and Drainage Paper, v. 56). 300 p.

Bodo, B. A., Unny, T. E. (1987). On the outputs of the stochsticized Nash-Dooge linear reservoir cascade. In: I. B. Macneill, G. J. Umphrey (Eds.), Stochastic hydrology, 1, 131-147. Dordretch: D. Reidel Publishing Company.

Burgess, T. M., Webster, R. (1980). Opitimal interpolation and isarithmic mapping of soil properties, 1 . The semivariogram and punctual kriging. Journal of Soil Science, 31, 315-331, DOI: 10.1111/j.1365-2389.1980.tb02084.x

Cambardella, C. A., Moorman, T. B., Novack, J. M., Parkin, T. B., Karlen, D. L., Turco R. F., Knopka, A. E. (1994). Field-scale variability of soil proprieties in central Iowa soils. Soil Science Society America Journal, 58, 1501-1511. DOI: $10.2136 /$ sssaj1994.03615995005800050033x

Centro de Pesquisas Meteorológicas e Climáticas Aplicadas a Agricultura - CEPAGRI. (2016). Clima dos Municípios Paulistas. Disponível em: <http://www.cpa.unicamp.br/ outras-informacoes/clima-dos-municipios-paulistas.html > . Acesso em: 16 mar. 2016.

Dagan, G. (2002). An overview of stochastic modeling of groundwater flow and transport: from theory to application. EOS - Transactions American Geophysical Union, 83, 621625. DOI: $10.1029 / 2002$ EO000421

Goovaerts, P. (1997). Geostatistics for natural resources evaluation. Oxford: Oxford University Press.

Hatch, C. E., Fisher, A. T., Revenaugh, J. S., Constantz, J., Ruehl, C. (2006). Quantifying surface water-groundwater interactions using time series analysis of streambed thermal records: method development. Water Resources Research, 42, W10410. DOI: 10.1029/2005WR004787

Heuvelink, G. B. M., Pebesma, E. J. (1999). Spatial aggregation and soil process modelling. Geoderma, 89, 47-65. DOI: 10.1016/S0016-7061(98)00077-9

Hipel, K. W., McLeod, A. I. (1994). Time series modelling of water resources and environmental systems. Amsterdam: Elsevier.

Hocking, M., Kelly, B. F. J. (2016). Groundwater recharge and time lag measurement through Vertosols using impulse response functions. Journal of Hydrology, 535, 22-35. DOI: 10.1016/j.jhydrol.2016.01.042

Instituto de Pesquisas Tecnológicas do Estado de São Paulo IPT. (1981). Mapa geológico do Estado de São Paulo. Escala 1:500.000. São Paulo: IPT.

Issaks, E. H., Srivastava, M. (1989). An introduction to applied geoestatistics. Nova York: Oxford University Press.

Jensen, M. E., Burman, R. D., Allen, R. G. (1990). Evapotranspiration and Irrigation Water Requirements. ASCE Manuals and Reports on Engineering Practice, v. 70. Nova York: American Society of Civil Engineers.

Johnston, K., Krivoruchko, K., Lucas, N., Ver Hoef, J. M. (2001). Using ArcGIS geostatistical analyst. Redlands: Esri.

Journel, A. G., Huijbregts, C. J. (1978). Mining geostatistics. Londres: Academic Press.

Knotters, M., Bierkens, M. F. P. (2000). Physical basis of time series models for water table depths. Water Resources Research, 36, 181-188. DOI: 10.1029/1999WR900288 
Manzione, R. L. (2014). Incorporação de incertezas associadas a predições de modelos hidrológicos aos instrumentos de gestão em recursos hídricos. Caderno Prudentino de Geografia, 36, 215-227.

Manzione, R. L., Knotters, M., Heuvelink, G. M. B. (2006). Mapping trends in water table depths in a Brazilian cerrado area. In: M. Caetano, M. Painho (Eds.), Procedings of Accuracy, 1, 449-458. Lisboa: Instituto Geográfico Português.

Manzione, R. L., Knotters, M., Heuvelink, G. M. B., Von Asmuth, J. R., Câmara, G. (2010). Transfer function-noise modeling and spatial interpolation to evaluate the risk of extreme (shallow) water-table levels in the Brazilian Cerrados. Hydrogeology Journal, 18, 1927-1937. DOI: 10.1007/s10040-010-0654-5

Manzione, R. L., Marcuzzo, F. F. N., Wendland, E. C. (2012). Integração de modelos espaciais e temporais para predições de níveis freáticos extremos. Pesquisa Agropecuária Brasileira, 47(9), 1368-1375. http://dx.doi.org/10.1590/ S0100-204X2012000900022

Manzione, R. L., Soldera, B. C., Wendland, E. (2017). Groundwater system response at sites with different agricultural land uses: case of the Guarani Aquifer outcrop area, Brotas/SP-Brazil. Hydrological Sciences Journal, 62, 28-35. DOI: 10.1080/02626667.2016.1154148

Melo, A. C. G., Durigan, G. (2011). Plano de manejo da Estação Ecológica de Santa Bárbara. São Paulo: Instituto Florestal.

Mestrinho, S. S. P. (2008). Monitoramento em água subterrânea. In: F. A. C. Feitosa, J. Manoel Filho, E. C. Feitosa, J. G. A. Demétrio (Eds.), Hidrogeologia: conceitos e aplicações, 1, 673-686. Rio de Janeiro: CPRM/LABHID.

Nash, J. E. (1958). Determining runoff from rainfall. Proceedings of Institute of Civil Engineer, 10, 163-184. DOI: 10.1680/iicep.1958.2025

Oliveira, J. B., Camargo, M. N., Rossi, M., Calderano Filho, B. (1999). Mapa Pedológico do Estado de São Paulo. Escala 1:500,000. Campinas: IAC/FAPESP.

Pachauri, R. K., Meyer, L. (2015). Climate change 2014: synthesis report. Geneva: Intergovernmental Panel on Climate Change - IPCC.

Pappenberger, F., Beven, K. J. (2006). Ignorance is bliss: or seven reasons not to use uncertainty analysis. Water Resources Research, 42, W05302. DOI: 10.1029/2005WR004820
Peterson, T. J., Western, A. W. (2014). Nonlinear time-series modeling of unconfined groundwater head. Water Resources Research, 50, 8330-8355. DOI: 10.1002/2013WR014800

Renard, P. (2007). Stochastic Hydrogeology: What Professionals Really Need? Ground Water, 45, 531-541. DOI: $10.1111 / j .1745-6584.2007 .00340 . x$

Ross, J. L. S., Moroz, I. C. (1996). Mapa geomorfológico do Estado de São Paulo. Revista do Departamento de Geografia, 10, 41-56. DOI: 10.7154/RDG.1996.0010.0004

Rubin, Y. (2004). Stochastic hydrogeology: challenges and misconceptions. Stochastic Environment Research and Risk Assessment, 18, 280-281. DOI: 10.1007/s00477-004-0193-5

Santarosa, L. V. (2016). Mapeamento de níveis freáticos do Sistema Aquifero Bauru (SAB) em área de proteção ambiental em Águas de Santa Bárbara/SP durante o ano hidrológico 2014/15. Dissertação (Mestrado). Botucatu: Faculdade de Ciências Agronômicas - UNESP.

Serviço Geológico do Brasil - CPRM. (2006). Domínio Geológico Litoestratográfico do Estado de São Paulo. Escala 1:750.000. São Paulo: CPRM.

Trangmar, B. B., Yost, R. S., Uehara, G. (1985). Applications of geostatistics to spatial studies of soil properties. Advances in Agronomy, 36, 45-94. DOI: 10.1016/S0065-2113(08)60673-2

Tucci, C. E. M. (2005). Modelos hidrológicos. $2^{\mathrm{a}}$ ed. Porto Alegre: Associação Brasileira de Recursos Hídricos/Editora da UFRGS.

Von Asmuth, J. R. (2012). Groundwater system identification through time series analysis. Tese (Doutorado em Hidrologia). Delft: TU Delft.

Von Asmuth, J. R., Bierkens, M. F. P. (2005). Modelling irregularly spaced residual series as a continuous stochastic process. Water Resources Research, 41, W12404. DOI: 10.1029/2004WR003726

Von Asmuth, J. R., Bierkens, M. F. P., Maas, C. (2002). Transfer function noise modelling in continuous time using predefined impulse response functions. Water Resources Research, 38, 23.1-23.12. DOI: 10.1029/2001WR001136

Von Asmuth, J. R., Knotters, M. (2004). Characterising groundwater dynamics based on a system identification approach. Journal of Hydrology, 296, 118-134. DOI: 10.1016/j.jhydrol.2004.03.015 
Von Asmuth, J. R., Maas, K., Bakker, M., Petersen, J. (2008). Modeling time series of ground water head fluctuation subjected to multiple stresses. Ground Water, 46, 30-40. DOI: $10.1111 / \mathrm{j} .1745-6584.2007 .00382 . \mathrm{x}$

Von Asmuth, J. R., Maas, K., Knotters, M., Bierkens, M. F. P., Bakker, M., Olsthoorn, T. N., Cirkel, D. G., Leunk, I., Schaars, F., Von Asmuth, D. C. (2012). Software for hydrogeologic time series analysis, interfacing data with physical insight. Environmental Modelling and Software, 38, 178-190. DOI: 10.1016/j.envsoft.2012.06.003
Yamamoto, J. K., Landim, P. M. B. (2013). Geoestatística: conceitos e aplicações. São Paulo: Oficina de Textos.

Yi, M., Lee, K. (2004). Transfer function-noise modelling of irregularly observed groundwater heads using precipitation data. Journal of Hydrology, 288, 272-287. DOI: 10.1016/j. jhydrol.2003.10.020

Zhang, Y. K., Zhang, D. (2004). Forum: The state of stochastic hydrology. Stochastic Environment Research and Risk Assessment, 18, 265. DOI: 10.1007/s00477-004-0190-8 